\section{Relação entre aterosclerose subclínica, pressão arterial e perfil lipídico em crianças e adolescentes obesos: uma revisão sistemática}

\author{
Relationship between subclinical atherosclerosis, blood pressure, and \\ lipid profile in obese children and adolescents: a systematic review
}

Juliana Pizzi ${ }^{12}$, Larissa Rosa da Silva ${ }^{2}$, Deise Moser $^{2}$, Neiva Leite ${ }^{3}$

\section{SUMÁRIO}

O estudo teve como objetivo revisar sistematicamente a literatura sobre espessamento médio-intimal (EMI), pressão arterial (PA) e perfil lipídico (PL) de crianças e adolescentes obesos e não obesos. A busca foi realizada em bases de dados eletrônicas (PubMed, Bireme e Elsevier ScienceDirect) entre 2000-2010. Os seguintes descritores, em inglês, foram usados: "obesity", "adolescents", "atherosclerosis" e "child", sendo utilizadas duas combinações: obesity+child+atherosclerosis e obesity+adolescents+atherosclerosis. A busca eletrônica inicial resultou em 3.211 artigos. Após a aplicação dos critérios de inclusão, 13 artigos foram selecionados. Destes, dois estudos demonstraram correlação significativa entre EMI e as variáveis PA, LDL e triglicerídeos. Nos outros estudos, não houve correlações significativas. Houve grande variabilidade metodológica entre os estudos. No entanto, obesos apresentaram maiores valores de EMI, PA e PL. Arq Bras Endocrinol Metab. 2013;57(1):1-6

\section{Descritores}

Crianças; adolescentes; aterosclerose; obesidade

\section{SUMMARY}

The present study aimed to systematically review the literature about intima-media thickness (IMT), blood pressure (BP), and lipid profile (LP) in obese and non-obese children and adolescents. The search was carried out in electronic databases (PubMed, Bireme, and Elsevier ScienceDirect) between 2000-2010. The following keywords, in English, were used: "obesity", "adolescents", "atherosclerosis" and "child", using two combinations: obesity+child+atherosclerosis and obesity+adolescents+atherosclerosis. The electronic search resulted in 3,211 manuscripts. After analysis of the inclusion criteria, 13 papers were selected. Of these, two studies showed significant correlation between IMT and the variables BP, LDL, and triglycerides. In other studies, no significant correlations were found. There is a wide methodological variability across the studies. However, obese children and adolescents had higher values of IMT, BP, and LP. Arq Bras Endocrinol Metab. 2013;57(1):1-6

\section{Keywords}

Children; adolescents; atherosclerosis; obesity
1 Universidade Paranaense (Unipar) Campus de Francisco Beltrão, Francisco Beltrão, PR, Brasil

${ }^{2}$ Núcleo de Qualidade de Vida, Curitiba, PR, Brasil ${ }^{3}$ Saúde da Criança e Adolescente, Universidade Federal do Paraná (UFPR), Núcleo de Pesquisa em Qualidade de Vida, Curitiba, PR, Brasil
Correspondência para:

Juliana Pizzi

Rua Videira, 67

85601-363 - Francisco Beltrão,

PR, Brasil

jupizzi@gmail.com

Recebido em 28/Jul/2011 Aceito em 27/Nov/2012

\section{INTRODUÇÃO}

$\mathrm{U}$ $\mathrm{m}$ estilo de vida ativo associa-se à menor incidência de várias doenças crônico-degenerativas e da mortalidade cardiovascular em adultos $(1,2)$, bem como a redução da prevalência da obesidade em crianças e adolescentes.

A obesidade infantil vem aumentando de forma significativa em muitos países, determinando várias complicações causadas por ela (3). Nos Estados Unidos, entre 1980 e 2004 a prevalência de obesidade aumentou de $15 \%$ para $33 \%$ entre a população adulta e a prevalência de sobrepeso em crianças aumentou de $6 \%$ para $19 \%$ (4). Atualmente, devido sua prevalência e seu alto custo financeiro e social, o excesso de gordura e peso corporal constitui um dos principais problemas de saúde pública (5). 
Estudos em crianças indicam que o processo da aterosclerose inicia-se em idades precoces e relaciona-se à obesidade e a fatores de risco cardiovasculares, como hipertensão arterial, dislipidemias e alterações do metabolismo da glicose; esta pode levar a síndrome metabólica, resistência à insulina, aumento do diabetes tipo 1 (6) e homocisteína (7), que contribuem para alterações na parede do vaso sanguíneo.

A disfunção endotelial arterial ou aterosclerose é uma anormalidade precoce durante a aterogênese e é considerada marcadora de dano arterial, que precede a formação da placa de gordura. A espessura médio-intimal da artéria carótida, que consiste na mensuração da espessura entre a túnica íntima e a túnica média da artéria por imagens de ultrassom, representa marcadora de aterosclerose subclínica (8). Estudos relacionam essa medida a fatores de risco vasculares, assim como relata a severidade e a extensão de doenças arteriais coronarianas, podendo predizer a probabilidade de eventos cardiovasculares em determinados grupos populacionais $(9,10)$.

O aumento da adiposidade corporal em indivíduos jovens favorece a elevação dos níveis pressóricos (11\% a $35 \%$ ) e alterações no perfil lipídico ( $40 \%$ a $60 \%$ ), podendo esses índices permanecerem até a idade adulta $(11,12)$. A associação desses fatores, juntamente com a disfunção endotelial, leva ao desenvolvimento da doença arterial coronariana, que é maior causa de morbimortalidade na vida adulta (13).

Nesse sentido, o objetivo desta revisão sistemática foi de verificar na literatura disponível os estudos que avaliaram o espessamento médio-intimal (EMI) como medida de aterosclerose subclínica e sua relação com os seguintes fatores de risco cardiovascular: pressão arterial (PA) e perfil lipídico em crianças obesas e não obesas, além de comparar essas variáveis na presença ou não de obesidade.

\section{MATERIAIS E MÉTODOS}

A revisão sistemática foi conduzida entre junho e agosto de 2010. A seleção dos descritores utilizados no processo de revisão foi efetuada mediante consulta aos DECs (descritores de assunto em ciências da saúde da Bireme). A busca foi realizada nas bases de dados eletrônicas do PubMed (www.pubmed.com), Bireme (www.bireme.br) e Elsevier (www.elsevier.com) (ScienceDirect), utilizando os seguintes descritores em língua inglesa e portuguesa: "obesity" (obesidade), "adoles- cents" (adolescentes), "atherosclerosis" (aterosclerose) e "child" (crianças), combinados em obesity+adolescents+atherosclerosis e obesity+child+atherosclerosis para localização nesta revisão, sem utilizar operadores lógicos como "AND", "OR" ou "AND NOT" para combinar os descritores e termos utilizados. Consideraram-se para efeito desta revisão as publicações no período de 2000 a 2010. Duas pesquisadoras efetuaram a busca nas três bases de dados selecionadas.

Por meio desse procedimento de busca, foram identificados inicialmente 3.211 estudos, sendo 1.256 para o descritor child e 681 para adolescents. Adotaram-se os seguintes critérios de inclusão: 1) somente artigos originais; 2 ) estudos realizados com crianças e/ou adolescentes; 3 ) estudos que realizaram avaliação da função endotelial; 4) estudos que apresentaram resumo; 5 ) estudos que avaliaram função endotelial por espessamento médio-intimal, sendo feita, inicialmente, a leitura dos resumos. Optou-se por não incluir teses, dissertações e monografias, visto que a realização de uma busca sistemática destas é inviável logisticamente.

Após a aplicação dos critérios citados, totalizaram-se 69 artigos na busca com o descritor child e 95 artigos na busca de adolescents, somando-se ao todo 164 artigos. Em seguida, aplicaram-se os critérios de exclusão: 6) estudos repetidos nas bases de dados e/ou duplicados nas duas buscas (child e adolescents); 7) não possuíam grupo de estudo com indivíduos obesos e não obesos/ou grupo controle; 8) fizeram intervenção e/ ou tratamento; 9) não avaliaram a pressão arterial e o perfil lipídico.

Os estudos incluídos nesta revisão foram analisados quanto à sua qualidade metodológica, segundo um protocolo de avaliação elaborado para este estudo, composto por sete critérios que os autores julgaram importantes, os quais foram considerados na discussão dos resultados de cada artigo. A pontuação de qualidade também foi realizada por dois investigadores de forma independente e dúvidas surgidas foram analisadas em reunião de consenso entre eles. Quanto maior a pontuação, melhor a qualidade do artigo. A escala foi composta pelos seguintes critérios: 1) seleção aleatória da amostra; 2 ) realização de cálculo amostral; 3 ) controle do estágio maturacional; 4) número maior que 40 indivíduos no grupo obeso; 5 ) exame de ultrassonografia de artéria carótida detalhado (posição do indivíduo, número máximo de medidas, local de posição do probe); 6) coeficiente inter e intraobservador; 7) análise estatística adequada. Os aspectos metodológicos podem ser observados na tabela 1 . 


\section{RESULTADOS}

$\mathrm{Na}$ tabela 2, são apresentadas informações gerais sobre os 13 estudos incluídos. Destes, nove foram publicados nos últimos cinco anos, três estudos foram publicados em periódicos classificados como Qualis Al e quatro estudos, no Qualis A2. Apenas três artigos utilizaram um delineamento transversal e três artigos, um delineamento de caso controle. Quanto à faixa etária, a composição das amostras variou, sendo que 10 artigos incluíram somente crianças e apenas dois artigos, somente adolescentes. A maioria dos estudos utilizou-se de hospital/clínica pediátrica para recrutar a amostra $(71,5 \%)$. Somente um artigo não deixou claro se passou por um Comitê de Ética.
O grupo de indivíduos obesos apresentou maiores valores de EMI (entre $0,38 \mathrm{~mm}$ e $0,74 \mathrm{~mm}$ ) do que o grupo dos controles (entre $0,35 \mathrm{~mm}$ e $0,57 \mathrm{~mm}$ ) em 10 estudos $(\mathrm{p}<0,05)$. Nos outros três artigos, os valores foram semelhantes entre os dois grupos. Na tabela 3, são apresentados as médias e os desvios-padrão da espessura médio-intimal e o nível de significância $(p)$.

Quanto à avaliação da PA, 11 artigos verificaram a diferença nos valores de pressão entre obesos e não obesos, e dois artigos não incluíram essa avaliação. Desses, cinco demonstraram valores maiores de pressão arterial sistólica (PAS) nos obesos quando comparados aos controles $(\mathrm{p}<0,05)(10,14-17)$. Para a pressão arterial diastólica (PAD), quatro artigos encontraram valores maiores nos não obesos em relação aos obesos $(\mathrm{p}<0,05)(8,9,10,16)$.

Tabela 1. Qualidade metodológica dos estudos incluídos na revisão

\begin{tabular}{|c|c|c|c|c|c|c|c|c|}
\hline Autor/Ano & 1 & 2 & 3 & 4 & 5 & 6 & 7 & Tota \\
\hline Morrison e cols., 2010 (14) & + & + & + & + & + & + & + & 7 \\
\hline Le e cols., 2010 (15) & + & - & - & + & + & + & + & 5 \\
\hline Mangge e cols., 2009 (16) & + & - & - & + & + & + & + & 5 \\
\hline Barja e cols., 2009 (10) & + & + & + & + & - & - & - & 4 \\
\hline Gil e cols., 2008 (17) & + & - & - & - & + & + & + & 4 \\
\hline Arnaíz e cols., 2008 (22) & + & - & + & + & + & + & + & 6 \\
\hline Demircioğlu e cols., 2008 (21) & + & - & - & + & + & + & + & 5 \\
\hline Beauloye e cols., 2007 (23) & + & - & + & + & + & + & + & 5 \\
\hline Arnaíz e cols., 2007 (18) & + & + & + & - & - & + & + & 5 \\
\hline Zhu e cols., 2006 (7) & + & - & - & + & + & + & + & 5 \\
\hline Zhu e cols., 2005 (8) & + & - & - & + & + & + & + & 5 \\
\hline Zhu e cols., 2005 (9) & + & - & - & + & + & + & + & 5 \\
\hline Woo e cols., (20) & + & + & + & - & + & + & + & 6 \\
\hline
\end{tabular}

Tabela 2. Artigos incluídos na revisão

\begin{tabular}{|c|c|c|c|c|}
\hline Autor/Ano & Periódico & Amostra & $\begin{array}{l}\text { Idade } \\
\text { (anos) }\end{array}$ & $\begin{array}{l}\text { Tipo de } \\
\text { estudo }\end{array}$ \\
\hline Morrison e cols., 2010 (14) & Atherosclerosis & 148 crianças, sendo 44 sobrepeso e 53 controle & $5-16$ & Transversal \\
\hline Le e cols., 2010 (15) & Journal of the AHA & 70 crianças, sendo 40 obesas e 30 com dislipidemia & $6-19$ & Longitudinal \\
\hline Mangge e cols., 2009 (16) & Atherosclerosis & 146 jovens, sendo 71 obesos e 75 controle & $9-16$ & Transversal \\
\hline Barja e cols., 2009 (10) & Revista Médica Chile & 209 crianças, sendo 114 obesos e 95 controle & $10-12$ & Longitudinal \\
\hline Gil e cols., 2008 (17) & Journal Korean Medical Science & 32 adolescentes, sendo 15 hipertensos e 17 controle & $13-18$ & Transversal \\
\hline Arnaíz e cols., 2008 (22) & International Journal of Cardiology & 103 crianças, sendo 45 eutróficos e 35 obesos & $6-16$ & Transversal \\
\hline Demircioğlu e cols., 2008 (21) & Journal Pediatric Gastroenterology Nutrition & 110 crianças, sendo 26 obesas e 30 controle & $8-17$ & Caso controle \\
\hline Beauloye e cols., 2007 (23) & $\begin{array}{c}\text { The Journal of Clinical Endocrinology \& } \\
\text { Metabolism }\end{array}$ & 197 crianças, sendo 104 obesas e 93 controle & $12-13$ & Transversal \\
\hline Arnaíz e cols., 2007 (18) & Revista Chilena de Pediatría & 83 crianças, sendo 26 obesos e 57 eutróficos & $8-11$ & Longitudinal \\
\hline Zhu e cols., 2006 (7) & European Journal of Pediatrics & 68 crianças, sendo 41 obesas e 27 controle & $10-13$ & Transversal \\
\hline Zhu e cols., 2005 (8) & European Journal of Pediatrics & 71 crianças, sendo 43 obesas e 28 controle & $7-14$ & Transversal \\
\hline Zhu e cols., 2005 (8) & European Journal of Pediatrics & 66 crianças, sendo 40 obesas e 26 controle & $7-14$ & Longitudinal \\
\hline Woo e cols., 2004 (20) & International Journal of Obesity & 72 crianças, sendo 36 obesas e 36 controle & $9-12$ & Caso controle \\
\hline
\end{tabular}


As médias máximas de PAS foram de $148 \mathrm{mmHg}$ e pressão arterial diastólica (PAD) de $73 \mathrm{mmHg}$, sendo que, neste estudo, o grupo dos obesos era também hipertenso. Os valores mínimos de PAS foram de $124 \mathrm{mmHg}$ e da PAD de $68 \mathrm{mmHg}$ no grupo dos obesos e, de $101 \mathrm{mmHg}$ de PAS e $55 \mathrm{mmHg}$ de PAD no grupo controle (Tabela 4).

Tabela 3. Média e desvio-padrão da espessura médio-intimal de cada estudo com o valor $p$

\begin{tabular}{|c|c|c|c|c|}
\hline \multicolumn{5}{|c|}{ Espessura médio-íntimal (EMI) (mm) } \\
\hline & Autor/Ano & Obesos & Controle & $\boldsymbol{p}$ \\
\hline 1 & Morrison e cols., 2010 (14) & $0,39(0,034)$ & $0,38(0,028)$ & $<0,05$ \\
\hline 2 & Le e cols., 2010 (15) & $0,53(0,05)$ & $0,53(0,05)$ & 0,07 \\
\hline 3 & Mangge e cols., 2009 (16) & $0,74(0,08)$ & $0,57(0,009)$ & $<0,001$ \\
\hline 4 & Barja e cols., 2009 (10) & $0,49(0,03)$ & $0,49(0,03)$ & ns \\
\hline 5 & Gil e cols., 2008 (17) & $0,62(0,013)$ & $0,50(0,01)$ & $<0,05$ \\
\hline 6 & Arnaíz e cols., 2008 (22) & $0,49(0,03)$ & $0,50(0,03)$ & 0,01 \\
\hline 7 & Demircioğlu e cols., 2008 (21) & $0,38(0,06)$ & $0,35(0,054)$ & 0,009 \\
\hline 8 & Beauloye e cols., 2007 (23) & $0,47(0,008)$ & $0,44(0,009)$ & 0,003 \\
\hline 9 & Arnaíz e cols., 2007 (18) & $0,49(0,03)$ & $0,50(0,03)$ & ns \\
\hline 10 & Zhu e cols., 2006 (7) & $0,61(0,11)$ & $0,46(0,05)$ & $<0,001$ \\
\hline 11 & Zhu e cols., 2005 (8) & $0,61(0,11)$ & $0,46(0,05)$ & $<0,001$ \\
\hline 12 & Zhu e cols., 2005 (9) & $0,61(0,11)$ & $0,46(0,11)$ & $<0,001$ \\
\hline 13 & Woo e cols., 2004 (20) & $0,49(0,04)$ & $0,45(0,04)$ & 0,03 \\
\hline
\end{tabular}

Tabela 4. Valores de pressão arterial e perfil lipídico para obesos e não obesos

\begin{tabular}{|c|c|c|c|c|c|c|}
\hline Artigo & Grupos & PAS/PAD & CT (mg/dL) & TG (mg/dL) & LDL (mg/dL) & HDL (mg/dL) \\
\hline \multirow[t]{2}{*}{1} & Obeso & $110 * / 67$ & $174 \pm 32,9^{*}$ & $117 \pm 79,7^{*}$ & $100,5 \pm 27,8^{*}$ & $49,5 \pm 9,7$ \\
\hline & Controle & $101 / 65$ & $154,7 \pm 22,8$ & $78,8 \pm 37,2$ & $78 \pm 17,9$ & $58,4 \pm 11,2^{*}$ \\
\hline \multirow[t]{2}{*}{2} & Obeso & $124^{*} / 66$ & $204 \pm 42^{*}$ & $187 \pm 121^{*}$ & $130 \pm 66^{\star}$ & $42 \pm 11$ \\
\hline & Controle & $120 / 65$ & $223 \pm 58$ & $152 \pm 105$ & $150 \pm 59$ & $46 \pm 13^{*}$ \\
\hline \multirow[t]{2}{*}{3} & Obeso & $120^{*} / 68$ & $162,4 \pm 25,1^{*}$ & $119,9 \pm 61^{*}$ & $51.9^{\Delta} \pm 14.2^{*}$ & 44 \\
\hline & Controle & $115 / 63^{*}$ & $151,9 \pm 28,6$ & $86,9 \pm 55,8$ & $32.4^{\Delta} \pm 11.0$ & $48^{\star}$ \\
\hline \multirow[t]{2}{*}{4} & Obeso & $109^{*} / 57$ & $155,4 \pm 28$ & $105,5 \pm 31,8^{*}$ & $88,4 \pm 23,5$ & $46 \pm 10,9$ \\
\hline & Controle & $102 / 55$ & $146,2 \pm 20$ & $55,4 \pm 19$ & $78 \pm 19$ & $57,3 \pm 10,3^{*}$ \\
\hline \multirow[t]{2}{*}{5} & Obeso & $148^{\star} / 73^{\#}$ & $176 \pm 25,4$ & $145,8 \pm 69,3$ & $102,6 \pm 25,6$ & $44,3 \pm 5,7$ \\
\hline & Controle & $116 / 72$ & $166,7 \pm 31,7$ & $168,3 \pm 118,4$ & $88,4 \pm 24,9$ & $44,6 \pm 11,5$ \\
\hline \multirow[t]{2}{*}{6} & Obeso & $107 / 57$ & $159,1 \pm 27,7$ & $94,7 \pm 46,2$ & $92,5 \pm 23,4$ & $47,7 \pm 10,9$ \\
\hline & Controle & $107 / 56$ & $143 \pm 23,5$ & $59,7 \pm 33,7$ & $75,2 \pm 19,4$ & $56,0 \pm 13,3$ \\
\hline \multirow[t]{2}{*}{7} & Obeso & & 172,5 & 106 & 100,5 & 49 \\
\hline & Controle & & 136 & 74,5 & 68,5 & 49,5 \\
\hline \multirow[t]{2}{*}{8} & Obeso & & 1544 & $72 \pm 4$ & $92 \pm 3$ & $48 \pm 1$ \\
\hline & Controle & & $153 \pm 3$ & $78 \pm 3$ & $83 \pm 3$ & $55 \pm 1$ \\
\hline \multirow[t]{2}{*}{9} & Obeso & $102 / 54$ & $152,8 \pm 28,9^{*}$ & $80,8 \pm 38,3^{*}$ & $87,9 \pm 25,6^{*}$ & $48,7 \pm 8,3$ \\
\hline & Controle & $101 / 55$ & $141,3 \pm 20$ & $60,6 \pm 25,3$ & $73,9 \pm 18$ & $55,3 \pm 10,9^{*}$ \\
\hline \multirow[t]{2}{*}{10} & Obeso & $120 / 73$ & $176,3 \pm 31^{*}$ & 150,41 & $86,2^{*}$ & 56,16 \\
\hline & Controle & $104 / 67^{*}$ & $124,9 \pm 20,5$ & 120,15 & 43,7 & $47,58^{\star}$ \\
\hline \multirow[t]{2}{*}{11} & Obeso & $120 / 73$ & $175,6 \pm 30,5^{\star}$ & $159,3 \pm 79,21$ & $92,4 \pm 33,5^{\star}$ & $56,9 \pm 16,4$ \\
\hline & Controle & $105 / 67^{\star}$ & $124,1 \pm 20,5$ & $124,6 \pm 40,9$ & $48,4 \pm 15,6$ & $47,2 \pm 9,4^{*}$ \\
\hline \multirow[t]{2}{*}{12} & Obeso & $118 / 73$ & $172,8 \pm 27,1^{*}$ & $162,9 \pm 81$ & $91,6 \pm 34,3^{*}$ & $54,6 \pm 16$ \\
\hline & Controle & $104 / 67^{\star}$ & $124,5 \pm 20,9$ & $124,6 \pm 42,7$ & $48,7 \pm 16$ & $47,6 \pm 9,4^{*}$ \\
\hline \multirow[t]{2}{*}{13} & Obeso & $105 / 64$ & $177,9 \pm 38,6$ & $115,1 \pm 44,3$ & $108,3 \pm 34,8$ & $47,6 \pm 12$ \\
\hline & Controle & $102 / 59$ & $177,9 \pm 34,8$ & $88,6 \pm 44,3$ & $112,1 \pm 30,9$ & $51,8 \pm 30,9$ \\
\hline
\end{tabular}

* Diferença estatística com p < 0,05; \# Grupo formado por crianças obesas e hipertensas; ${ }^{\triangle}$ LDL oxidada; PAS: pressão arterial sistólica; PAD: pressão arterial diastólica; CT: colesterol total; TG: triglicerídeos; LDL: lipoproteína de baixa densidade; HDL: lipoproteína de alta densidade. 
Em relação ao perfil lipídico, sete estudos demonstraram valores maiores de $\mathrm{CT}$ nos obesos do que nos não obesos $(\mathrm{p}<0,05)(7-9,14-16,18)$. Em relação aos TG, cinco artigos encontraram diferença entre os grupos, sendo maiores nos obesos $(\mathrm{p}<0,05)(10,14,15,16,18)$. A variável LDL foi maior nos obesos em sete estudos analisados quando comparados aos controles $(\mathrm{p}<0,05)$ (7-9,14-16,18). No entanto, o HDL apresentou-se mais elevado nos não obesos do que nos obesos em 8 estudos $(\mathrm{p}<0,05)(7-10,14-16,18)$.

Com relação às correlações entre EMI, dos 13 estudos, apenas dois estudos encontraram correlação significativa entre EMI e PA, LDL e TG.

\section{DISCUSSÃO}

A obesidade infantojuvenil está relacionada com maior risco de doenças cardiovasculares no futuro. Essa condição tem atraído significativa atenção científica, pois o início de eventos que agridem o endotélio vascular pode correr na infância, porém, sinais clínicos da aterosclerose geralmente só aparecem na fase adulta (19). Este estudo buscou fazer uma revisão sistemática quanto às diferenças entre o EMI em crianças e adolescentes obesos e não obesos, e sua relação com valores de pressão arterial e níveis de perfil lipídico.

Nesta análise, foi observado que os valores médios de EMI apareceram no grupo de obesos, após comparação entre os grupos, sendo que o maior valor médio foi de $0,74( \pm 0,08) \mathrm{mm}(16)$ e um menor valor médio, de $0,49( \pm 0,05) \mathrm{mm}(20)$. Já no grupo não obeso, os valores médios maiores e menores observados foram, respectivamente, $0,57( \pm 0,09) \mathrm{mm}(16) \mathrm{e}$ $0,38( \pm 0,009) \mathrm{mm}(14)$. Esses resultados discordantes, identificados pela diferença de $0,25 \mathrm{~mm}$, possivelmente são explicados pelas diferentes metodologias empregadas para mensuração do EMI e uma variação entre os avaliadores. Nesse contexto, nove estudos fizeram a medição de ambos os lados (direito e esquerdo), sete estudos apresentaram um dos coeficientes de variação (intraobservador, interobservador ou os dois coeficientes), o número de mensurações por segmento de artéria carótida e o ponto de obtenção da medida de EMI também variou entre as pesquisas analisadas. Entretanto, apesar das diferenças metodológicas, a maioria dos estudos (nove estudos) demonstrou valores maiores de EMI no grupo de obesos. Isso mostra forte ligação entre a obesidade e as alterações estruturais nas artérias em população pediátrica, relacionadas principalmen- te a um perfil lipídico desfavorável (20). O processo aterosclerótico é diretamente influenciado pelo grau de obesidade e níveis de lipídeos séricos de crianças e adolescentes (21).

Testes não invasivos, tais como a ultrassonografia de onda-B da artéria carótida, vêm tornando-se método potencial para medir e monitorar a aterosclerose $(21,17)$, visto que são tecnicamente fáceis de manusear, confiáveis, relativamente baratos e isentos de radiação (15).

Além dos exames de sangue para perfil lipídico (12), são importantes as dosagens dos marcadores inflamatórios, como adiponectina e proteína C-reativa (PCR) $(14,22,23)$, pois apresentaram alterações nos obesos em alguns estudos analisados, se comparados ao grupo controle, indicando presença de inflamação nesses indivíduos.

Destaca-se ainda que a idade cronológica influencia no resultado da medição do EMI $(14,15)$, podendo variar pela presença de fatores de risco cardiovasculares únicos ou múltiplos, por fatores genéticos ou riscos não documentados $(10,18)$. As crianças podem estar em diferentes etapas do processo da aterosclerose de acordo com sua idade, ou seja, as chances de essa interação aparecer são maiores em crianças com idades superiores (18).

A hipertensão é um dos principais fatores para o desenvolvimento da aterosclerose, chamada de disfunção endotelial na infância $(17,23)$. No Brasil, estima-se que essa enfermidade afete cerca de $20 \%$ a $30 \%$ dos adultos (24) e de $1 \%$ a $13 \%$ da população infantojuvenil (25).

De acordo com esse levantamento, identificou-se carência de estudos científicos sobre essas relações fisiológicas nesta faixa etária, tanto em termos mundiais quanto no Brasil, já que não foi encontrado nenhum estudo, especificamente, com essa relação de EMI, pressão arterial e perfil lipídico de crianças e adolescentes. Esses estudos são importantes em decorrência da relação entre a obesidade e o aumento da espessura médio-intimal, visto que a prevalência de excesso de peso no Brasil entre meninos e rapazes de 10 a 19 anos de idade de $3,7 \%$ (1974-75) foi para $21 \%$ (2008-09), e entre as meninas e moças foi de 7,6\% para 19,4\% (26). Confirmado pelos artigos estudados, adolescentes obesos têm maior risco de apresentarem sinais de aterosclerose precocemente e, logo, estão mais suscetíveis a desenvolverem doenças cardiovasculares na vida adulta.

Em conclusão, com base na compilação realizada, é possível investigar riscos cardiovasculares em crianças e adolescentes, principalmente naqueles que apresentam obesidade, por exames da espessura médio-intimal das 
artérias, devido à forte associação da EMI em crianças obesas com pressão arterial, tanto sistólica como diastólica, e com as variáveis do perfil lipídico (colesterol total, LDL, HDL, triglicerídeos, glicose e insulina). Entretanto, a maioria não obteve significância estatística suficiente por diferentes razões: o intervalo de idade era abrangente, o que pode associar-se a importantes mudanças fisiológicas pelo estágio maturacional; as medidas dos segmentos da artéria carótida não foram específicas e padronizadas, e alguns métodos podem não ter sido eficazes para encontrar as diferenças estatísticas demonstradas em outros estudos.

Hábitos sedentários associados a um estilo de vida pouco ativo têm sido uma crescente em todo o mundo. Adultos e crianças alimentam-se, principalmente, de comidas pouco nutritivas e ricas em gordura, resultando em uma epidemia de obesidade. Com isso, a associação dos fatores de risco cardiovasculares com os processos de alterações estruturais e disfunções endoteliais implica grande importância. Logo, ressalta-se a necessidade de mais estudos científicos sobre essas relações na população mundial e, especificamente, na população brasileira, para que haja um melhor planejamento e posterior intervenção, objetivando a prevenção das doenças cardiovasculares na fase adulta.

Fontes de financiamento: o presente estudo não teve fontes de financiamento externas.

Vinculação acadêmica: este artigo é parte da dissertação de Mestrado de Juliana Pizzi pelo Programa de Pós-graduação em Educação Física da Universidade Federal do Paraná (UFPR).

Agradecimentos: ao Conselho Nacional de Desenvolvimento Científico e Tecnológico (CNPq).

Declaração: os autores declaram não haver conflitos de interesse científico neste estudo.

\section{REFERÊNCIAS}

1. Lazzolli JK, Nobrega ACL, Carvalho T, Oliveira MAB, Teixeira JAC, Leitão MB, et al. Atividade física e saúde na infância e adolescência: posicionamento oficial. Rev Bras Med Esporte. 1998;4(4):107-9.

2. Mello ED, Luft VC, Meyer F. Obesidade infantil: como podemos ser eficazes? J Pediatr. 2004;80(3):173-82.

3. Bergmann MLA, Halpern R, Bergmann GG. Perfil lipídico, de aptidão cardiorrespiratória, e de composição corporal de uma amostra de escolares de $8^{\mathrm{a}}$ série de Canoas/RS. Rev Bras Med Esporte. 2008;14(1):22-7.

4. Celemajer DS. Endothelial dysfunction: does it matter? Is it reversible? J Am Coll Cardiol. 1997;30(2):325-33.

5. Guedes DP, Guedes JE. Controle do peso corporal: composição corporal, atividade física e nutrição. Londrina: Midiograf; 1998. p. 11.

6. Ogden CL, Yanovski SZ, Carroll MD, Flegal KM. The epidemiology of obesity. Gastroenterology. 2007;132:2087-102.

7. Zhu W, Huang X, He J, Li M, Neubauer H. Elevated plasma homocysteine in obese schoolchildren with early atherosclerosis. Eur J Pediatri. 2006;165(5):326-31.

8. Zhu W, Huang X, He J, Li M, Neubauer H. Association of hyperviscosity and subclinical atherosclerosis in obese schoolchildren. Eur J Pediatr. 2005;164(10):639-45.
9. Zhu W, Huang X, He J, Li M, Neubauer H. Arterial intima-media thickening and endothelial dysfunction in obese Chinese children. Eur J Pediatr. 2005;164(6):337-44.

10. Barja S, Acevedo M, Arnaiz P, Berríos X, Bambs C, Guzmán B, et al. Marcadores de aterosclerosis temprana y síndrome metabólico en niños. Rev Méd Chile. 2009;137(4):522-30.

11. Sorof JM, Lai D, Turner J, Poffenbarger J, Portman R J. Overweight, ethnicity and the prevalence of hypertension in schoolaged children. Pediatrics. 2004;113(3):475-82.

12. Sullivan CS, Beste J, Cummings DM, Hester VH, Holbrook T, Kolasa KM, et al. Prevalence of hyperinsulinemia and clinical correlates in overweight children referred for lifestyle intervention. $\mathrm{J}$ Am Diet Assoc. 2004;104(3):433-6.

13. Hayman LL, Williams CL, Daniels SR, Steinberger J, Paridon $\mathrm{S}$, Dennison BA, et al.; Committee on Atherosclerosis, Hypertension, and Obesity in Youth (AHOY) of the Council on Cardiovascular Disease in the Young, American Heart Association. Cardiovascular health promotion in the schools: a statement for health and education professionals and child health advocates from the Committee on Atherosclerosis, Hypertension, and Obesity in Youth (AHOY) of the Council on Cardiovascular Disease in the Young, American Heart Association. Circulation. 2004;110(15):2266-75.

14. Morrison KM, Dyal L, Conner W, Helden E, Newkirk S, Yusuf $\mathrm{S}$, et al. Cardiovascular risk factors and non-invasive assessment of subclinical atherosclerosis in youth. Atherosclerosis. 2010;208(2):501-5.

15. Le J, Zhang D, Menees S, Chen J, Raghuveer G. "Vascular age" is advanced in children with atherosclerosis-promoting risk factors. Circulation. 2010;3:8-14.

16. Mangee H, Almer G, Haj-Yahya S, Grandits N, Gasser R, et al. Nuchal thickness of subcutaneous adipose tissue is tightly associated with na increased LMW/total adiponectin ratio in obese juveniles. Atherosclerosis. 2009;203(1):277-83.

17 GilTY, Sung CY, Sung SS, Hong YM. Intima-media thickness and pulse wave velocity in hypertensive adolescents. J Korean Med Sci. 2008;23(1):35-40.

18. Arnaíz $P$, Acevedo MB, Barja $S$, Berríos $X$, et al. Arteriosclerosis subclínica, factores de riesgo cardiovascular clásicos e emergentes em niños obesos chilenos. Rev Chil Pediatr. 2007;78(2):135-42.

19. Rossetti MB, Britto RR, Norton RC. Prevenção primária de doenças cardiovasculares na obesidade infantojuvenil: efeito anti-inflamatório do exercício físico. Rev Bras Med Esporte. 2009;15(6):472-5.

20. Woo KS, Chook P,Yu CW, Sung RY, Qiao M, Leung SS, et al. Overweight in children is associated with arterial endothelial dysfunction and intima-medias thickening. Int J Obes Relat Metab Disord. 2004;28(7):852-7.

21. Demircioğlu F, Koçyiğit A, Arslan N, Cakmakçi H, Hizli S, Sedat AT. Intima-media thickness of carotid artery and susceptibility to atherosclerosis in obese children with nonalcoholic fatty liver disease. J Pediatr Gastroenterol Nutr. 2008;47(1):68-75.

22. Arnaiz $P$, Acevedo M, Barja S, Aglony M, Guzmán B, Cassis B, et al. Adiponectin levels, cardiometabolic risk factors and markers of subclinical atherosclerosis in children. Int $\mathrm{J}$ Cardiol. 2010;138(2):138-44.

23. Beauloye V, Zech F, Mong TT, Clapuyt P, Maes M, Brichard SM. Determinants of early atherosclerosis in obese children and adolescents. J Clin Endocrinol Metab. 2007;92(8):3025-32.

24. Simonetti JP, Batista L, Carvalho LR. Hábitos de saúde e fatores de risco em pacientes hipertensos. Rev Latinoam Enferm. 2002;10:415-22.

25. Sociedade Brasileira de Hipertensão. V Diretrizes Brasileiras de Hipertensão Arterial. Arq Bras Cardiol. 2007;89(3):24-79.

26. Instituto Brasileiro de Geografia e Estatística - IBGE. Disponível em: http://www.ibge.gov.br/home/presidencia/noticias/noticia_visualiza.php?id_noticia=1699\&id_pagina=1>. Acesso em: 12 jun, 2011. 